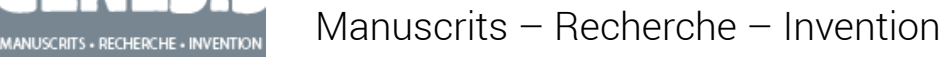

$30 \mid 2010$

Théorie : état des lieux

\title{
Pour une didactique du prérédactionnel
}

\section{Olivier Lumbroso}

\section{OpenEdition}

Journals

Édition électronique

URL : https://journals.openedition.org/genesis/135

DOI : 10.4000/genesis. 135

ISSN : 2268-1590

\section{Éditeur :}

Presses universitaires de Paris Sorbonne (PUPS), Société internationale de génétique artistique littéraire et scientifique (SIGALES)

\section{Édition imprimée}

Date de publication : 20 juin 2010

Pagination : 177-184

ISBN : 978-2-84050-697-3

ISSN : 1167-5101

\section{Référence électronique}

Olivier Lumbroso, «Pour une didactique du prérédactionnel », Genesis [En ligne], 30 | 2010, mis en ligne le 30 mai 2012, consulté le 30 mars 2023. URL : http://journals.openedition.org/genesis/135 ; DOI : https://doi.org/10.4000/genesis.135 


\title{
Pour une didactique du prérédactionnel
}

\author{
Olivier Lumbroso
}

Décidément, il faut leur apprendre à « littératurer ».

Almuth GrÉsILLON ${ }^{1}$

E n étudiant les processus de la création dans les manuscrits d'écrivains, dans les dessins d'architectes ou les carnets de scientifiques, la critique génétique a fait la preuve de son ouverture interdisciplinaire ${ }^{2}$. Cet article souhaite poursuivre cet enrichissement transversal en étudiant quelques interactions possibles entre la génétique et la didactique de l'écriture, avec l'objectif suivant : montrer que, transférée dans le domaine des apprentissages, l'écriture dite « à programme ${ }^{3}$ », théorisée par la critique génétique, peut enrichir les pratiques scripturales des élèves. En effet, elle incite à porter un regard plus appuyé sur les documents de travail «prérédactionnels », qui précèdent le brouillon rédigé dont les démarches d'enseignement ont fait, aujourd'hui, l'une des clefs de voûte du projet d'écriture.

\section{Brouillon scolaire et types d'écriture}

Dès l'école primaire, le chantier d'écriture qu'élabore un enseignant peut s'inscrire peu ou prou dans un programme didactique à dimensions multiples. C'est un dispositif qui aide l'élève à se représenter les moyens qui lui permettent de réaliser la tâche demandée. Il se caractérise souvent par une étape initiale de négociation du projet définissant, en classe, les buts à atteindre, les modalités de travail, les consignes et les règles d'écriture. Son déroulement suppose aussi des phases de réécriture dans le brouillon, étayées par des outils et des activités parallèles, qui intègrent des apprentissages sur les textes et la langue.
Ce dispositif reflète les avancées de la didactique des vingt dernières années. Cet article bref ne recense pas, faute de place, ses apports qui s'appuient sur des travaux importants, dont il faudrait dresser l'historique et la bibliographie, mais il est possible toutefois de rappeler leur esprit général ${ }^{4}$. Ils ont mis en évidence la complexité

1. A. Grésillon, «Les manuscrits littéraires : le texte dans tous ses états », Pratiques, $n^{\circ}$ 57, 1988, p. 107-122. Queneau invente le mot « Littératurer » dans Le Chiendent (1956).

2. Voir les numéros de Genesis sur l'architecture ( $\left.n^{\circ} 14,2000\right)$ et l'écriture scientifique $\left(\mathrm{n}^{\circ} 20,2003\right)$. Voir encore l'article de P.-M. de Biasi, "Génétique des arts ", dans Critique génétique. Concepts, méthodes, outils, dir. O. Anokhina et S. Pétillon, Paris, IMEC, coll. «Inventaires », 2009.

3. L. Hay, « La troisième dimension de la littérature », Texte, $n^{\circ} 5 / 6$, 1986-1987. De façon souple, la critique génétique a distingué deux grandes tendances d'écriture, d'ailleurs souvent mêlées : d'un côté, les écrivains qui, comme Zola dans ses dossiers préparatoires, s'appuient sur des manuscrits de travail avant de rédiger, relevant de ce que Louis Hay nomme écriture « à programme » et ceux qui, de l'autre, en se passant de conception préliminaire, entrent in medias res dans l'écriture dite « à processus ». P.-M. de Biasi a repensé ces deux tendances en distinguant écriture à « structuration rédactionnelle » et écriture à « programmation scénarique », dans La Génétique des textes, Paris, Nathan, 2000, p. 33. Louis Aragon, refusant l'image du romancier à programme, « espèce d'ingénieur, qui sait fort bien où il veut en venir, résout des problèmes dont il connaît le but », avoue n'avoir jamais écrit une histoire dont il connaissait à l'avance le déroulement, et poursuit, «j'ai toujours été, écrivant, comme un lecteur qui fait la connaissance d'un paysage ou de personnages dont il découvre le caractère, la biographie, la destinée », Je n'ai jamais appris à écrire ou les Incipits, Paris, Flammarion, coll. «Champs », 1981, p. 56.

4. Voir la note de synthèse de Bertrand Daunay, «État des recherches en didactique de la littérature », Revue française de pédagogie, $\mathrm{n}^{\circ}$ 159, 2007, p. 139-189 
de l'acte d'écrire, et ont orienté les propositions d'apprentissage afin que l'élève, dès son plus jeune âge, entre dans une pratique scripturale étayée par l'enseignant, et que soit stimulée son envie d'écrire et de réécrire, au sein de projets socialisés faisant sens. L'apport didactique fondamental réside certainement dans l'idée que c'est en écrivant qu'on apprend à écrire, aux antipodes des deux logiques qui consistaient à penser qu'il faut apprendre à écrire avant d'écrire (imitation de modèles au début du $\mathrm{Xx}^{\mathrm{e}}$ siècle) ou qu'il faut apprendre à écrire après avoir écrit (l'expression libre des années soixante-dix). C'est pourquoi le brouillon textualisé occupe, à juste titre, une place si privilégiée dans les approches modernes de l'écriture scolaire. Toutefois, c'est aussi, semble-t-il, un outil qui possède ses limites.

En effet, au sein du cadre didactique précédemment décrit, l'élève va prendre son cahier de brouillon puis va «entrer en textualisation»: il se jette dans une forme d'écriture à processus. Cet acte, pour lui, est souvent une habitude naturelle. Or, il s'agit bien d'un modèle culturel possible, comme nous l'apprend la critique génétique. Malgré tout, il s'est imposé dans nos pratiques scolaires, notamment pour aborder les genres narratifs. L'idée persiste que, pour écrire un récit, il suffit de travailler « au fil de la plume », tandis que pour bâtir une dissertation convaincante, il est nécessaire d'échafauder d'abord un plan qui en détaille le circuit argumentatif. L'imagination souveraine et la fantaisie pour le récit d'aventure, les lois sévères de la rhétorique et de la raison pour l'essai. Les enseignants confessent pourtant la faible efficacité de ces brouillons rédigés du texte narratif, du point de vue de la révision : les scripteurs débutants font le toilettage orthographique en surface, mais ils ne parviennent pas à le modifier au-delà. Des raisons l'expliquent : insérer précocement le brouillon dans le tissu syntaxique de la phrase, du paragraphe et du texte, revient, pour beaucoup d'élèves, à demander à un maçon de cimenter un mur avant d'en avoir agencé les briques. Pour un jeune scripteur, les modifications seront coûteuses dans un brouillon rédigé où il faudra refondre, au moyen de suppressions, de déplacements ou d'ajouts, les ligatures logico-temporelles qui ont fixé, parfois figé, les structures sémantiques et pragmatiques. Signe que le raturage dans le brouillon textualisé ne peut pas tout accomplir : si l'élève est capable de révision à l'échelle du mot ou de la phrase, il lui est difficile, par ce seul moyen, d'envisager les rapports entre la partie et le tout, la rature locale et le sens global, la microstructure de la phrase et la macrostructure du discours. En partant de la description de ces pratiques scripturales, l'hypothèse peut être faite que la textualisation précoce du brouillon constitue un maillage morpho-syntaxique qui peut limiter les dynamiques profondes de la réécriture chez un élève devant tout à la fois répondre aux consignes du sujet, inventer, construire et réviser, dans l'action même de formuler son discours. Afin de proposer des solutions à ces apories, d'autres formes d'écrits préparatoires, inspirées par les avant-textes d'écrivains relevant de l'écriture à programmation scénarique, sont susceptibles de transferts scolaires qui permettraient de repenser les relations de la mise en texte et de la planification. Justement, les écritures à programme proposent des outils susceptibles d'apporter des solutions. Écrire un canevas préalable, dresser un plan général du récit, rédiger des fiches sur les personnages, dessiner les croquis des lieux fictionnels, se donner des autoconsignes d'écriture constituent autant de moyens pour déplier l'acte de création, le décomposer en éléments constitutifs au sein d'un programme souple qui les fait interagir. De plus, ces écrits peuvent assurer le passage d'une échelle à l'autre (le plan), d'un type de texte à un autre (le croquis, la fiche), d'une visée à une autre (une note documentaire qui informe, un canevas qui raconte)...

Un chantier se dessine donc : celui d'une didactique $d u$ prérédactionnel qui se demanderait notamment comment l'écriture à programme peut enrichir les pratiques scripturales des élèves, à des niveaux de classe et dans des projets différents. Écrire un récit ne serait plus dérouler une histoire au fil de la plume mais établir des itinéraires, plus ou moins denses selon l'âge des scripteurs, entre des espaces de construction intermédiaires et en interaction. La recherche didactique devra sans doute dresser les typologies de programmes d'écriture possibles et, selon les classes et leurs besoins, selon les profils de scripteurs et leurs stratégies, évaluer l'efficacité de ces apprentissages. Elle pourra aussi décrire les enjeux cognitifs, riches et variés, qui se dégageront du circuit génétique construit par l'élève : le croquis de la main d'un enfant de Cours Moyen, qui aborde la production verbale par le détour 


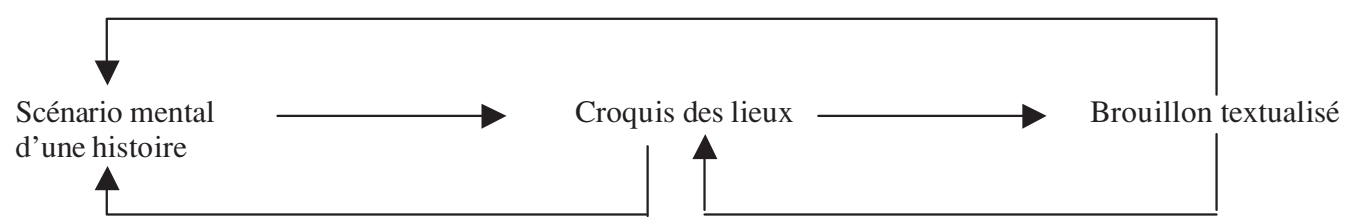

Niveau école (CM1-CM2) : programme court (croquis/brouillon)

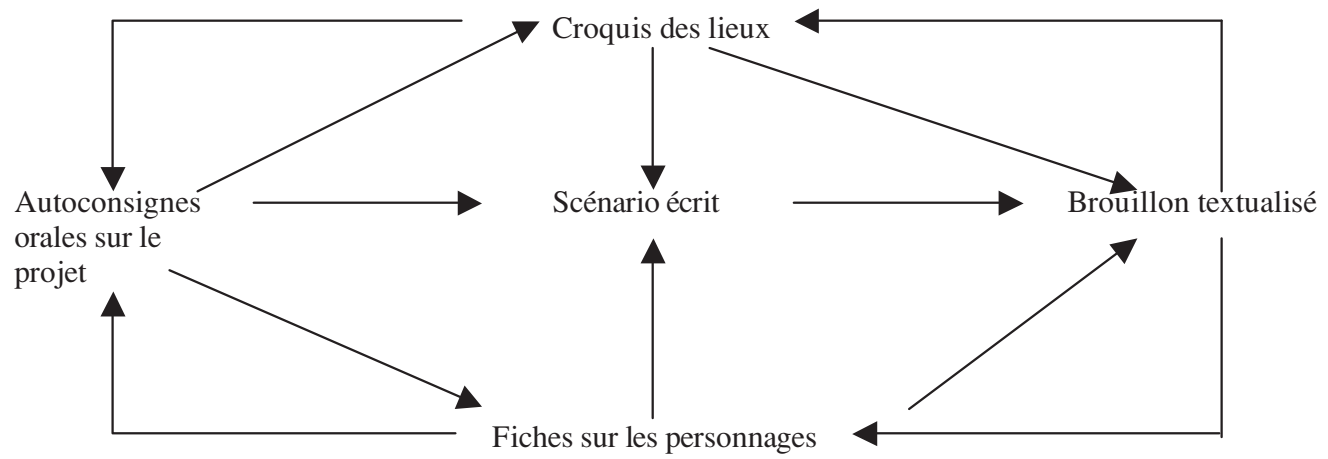

Niveau collège $\left(3^{e}\right)$ : programme long (autoconsignes orales/scénario/croquis/fiches/brouillon)

du non-verbal procède d'une démarche très différente des consignes d'écriture que ce même écolier ou qu'un collégien se donnerait à lui-même, entrant ainsi dans une posture réflexive d'autocommentaire. Voici quelques exemples d'écritures à programme, dont certaines ont été testées dans des classes 5 (voir schéma ci-dessus).

L'expérience qui suit, réalisée dans une classe de lycée, transfère avec d'autres objectifs le programme long du collège.

\section{Écriture à programme et contexte scolaire}

Afin d'illustrer ces schémas théoriques, une expérience réalisée en lycée peut être brièvement exposée. Elle porte sur l'usage d'autoconsignes au sein d'un scénario métatextuel, qui assiste l'écriture d'une nouvelle en seconde 6 .

L'autoconsigne est fréquente dans les avant-textes des écrivains. Dans le Journal des Faux-Monnayeurs, Gide note ses réflexions et ses difficultés : «[...] je voudrais pourtant éviter ce qu'a d'artificielle une "intrigue" ; mais il faudrait que les événements se groupent indépendamment de Lafcadio et pour ainsi dire : à son insu7. » La rhétorique volontariste d'un Zola qui se prescrit à lui-même ce qu'il faut chercher, écrire ou ne pas oublier multiplie aussi les consignes qui guident la fabrication de l'œuvre :

Je voudrais, après Le Rêve, faire un roman tout autre; d'abord dans le monde réel ; puis sans description, sans art visible, sans effort, écrit d'une plume courante ; du récit simplement ; et comme sujet, un drame violent à donner cauchemar à tout Paris $[\ldots]^{8}$.

5. Voir notre article : «Esquisse d'un dialogue entre didactique de l'écrit et critique génétique : l'élève "auteur-dessinateur" ", Revue française de pédagogie, $\mathrm{n}^{\circ} 159$, mai-juin 2007.

6. Classe de seconde du lycée Rabelais de Saint-Brieuc. Le sujet d'invention était le suivant : «Un personnage d'aujourd'hui se trouve dans un lieu et regarde un objet emblématique de ce lieu. Le même objet est présent à la fin de l'histoire. Qu'est-il devenu ? Dans quel lieu ? Vu par qui ? Pourquoi ? » Depuis les travaux de Hayes et Flower sur les processus rédactionnels, la pyscholinguistique s'est souvent penchée sur la planification et la révision, à travers l'étude des «protocoles verbaux », que nous inscrivons ici, sous la désignation d' "autoconsignes », dans le contexte d'une vie de classe (voir bibliographie).

7. A. Gide, Journal des Faux-Monnayeurs, Paris, Gallimard, coll. «L'Imaginaire », 1995, p. 76.

8. Ébauche de La Bête humaine, BnF, ms., nafr $10247, \mathrm{f}^{\circ} 338$. 
En tant que soliloque programmatique ou acte de langage indirect à caractère prescriptif, l'ébauche relève bien de la visée injonctive ${ }^{9}$. Toutefois, celle-ci s'assouplit selon les hésitations du romancier. Les expressions évaluatives de Zola comme «cela est mauvais », «non », s'accompagnent d'une échelle de modalisation qui va de la certitude au doute, du nécessaire au possible. Le manuscrit s'apparente ainsi à un laboratoire expérimental de choix évolutifs. Or, chez l'élève, rares sont les traces métatextualisées, écrites ou orales, de ce qu'il veut faire et ne veut plus faire, croit faire et sait faire, pas de « gueuloir » ou de «parole intérieure extériorisée » selon la formule d'Almuth Grésillon ${ }^{10}$. À côté de la grille de l'évaluateur ne faudrait-il pas dès lors introduire légitimement la voix de l'élève auto-évaluateur, programmant sa stratégie de lecture du sujet et ses choix tactiques d'écriture ? Nombreuses sont les études génétiques qui ont insisté sur le caractère polyphonique des avant-textes d'écrivains et sur la « double locution génétique » (J.-L. Lebrave), grâce à laquelle le scripteur se fait lecteur critique de lui-même. Cette compétence métatextuelle, qui dépasse la seule révision du brouillon rédigé, introduit l'élève au cœur de son aventure scripturale, à la fois réflexive et en devenir. La discussion avec soi-même, ou en classe, autour de problèmes d'écriture, constitue un métadiscours d'une grande créativité, dont l'approche peut s'avérer, en plus, très ludique.

Les lycéens, ravis d'entrer dans une autre pratique, ont produit la nouvelle sur un « carnet de bord », où la page de gauche était consacrée au métalangage et la page de droite au brouillon rédigé. Très naturellement, et sans avoir eu connaissance de quelconques manuscrits d'écrivain qui auraient pu aboutir à des pastiches génétiques, ils ont produit les consignes métatextuelles dont ils avaient besoin et qui, pour la grande majorité, les ont aidés à poser et à résoudre des problèmes de poétique narrative dont ils n'avaient pas conscience avant :

Autoconsignes descriptives : l'élève (se) dit ce qu'il fait/ ne fait pas. Il raconte son action créatrice :

Mélanie : «Je reprends tout ce qui est déjà écrit car trop compliqué. // Je supprime ce passage car je trouve qu'il n'a finalement pas beaucoup d'intérêt et qu'il complique. »
Autoconsignes prescriptives: l'élève (se) dit ce qu'il doit faire/ne doit pas faire. Il se donne une action à accomplir :

Sarah: «Je dois préciser les lieux sinon les "lecteurs" auront du mal à comprendre où se passent les scènes. »

Autoconsignes explicatives : l'élève (se) justifie ses choix, analyse une hésitation, explique la raison d'une action :

Camille: «J'ai un "bon" début mais pour la suite, je sèche. Je veux que ça aille vers la gaieté pour changer, que l'histoire se finisse bien ; ce serait trop triste autrement. »

Sarah: «Pour l'objet, j'avais pensé à une bague, mais la bague est un objet trop utilisé que ce soit dans le Seigneur des Anneaux ou encore Narnia : le neveu du magicien. »

Autoconsignes narratives : l'élève scénarise son histoire :

Gladys : « À la fin de l'histoire, il va finir par lui rendre sa liberté même s'il était très attaché à cet oiseau. »

Les élèves se sont exprimés sur cette méthode d'écriture, produisant les traces matérielles d'un soliloque intérieur. Même s'il en ressort parfois une gêne devant une pratique qu'il faut s'approprier dans le temps, nombreux sont ceux qui y perçoivent d'ores et déjà des intérêts : démarrer et se donner des buts à soi-même, faire un plan assurant un déblocage, poser ses idées et faire des choix narratifs. Ainsi le témoignage de Camille, sensible, comme Paul Valéry, aux carrefours de possibles que dévoile le manuscrit de travail : « Je notais tout ce que je pensais, surtout au début. Moi, qui n'avais pas d'idées, ça me permettait d'avancer tout de même. Tout ce qui me passait par la tête je l'écrivais, ouvrant les possibilités, en fermant d'autres. »

9. Voir H. Mitterand, «Le méta-texte génétique dans les Ébauches de Zola », Genesis, n 6, « Enjeux critiques », 1994, p. 47-60; pour une étude des autoconsignes génétiques de Zola, voir aussi l'essai synthétique $L e$ Signe et la consigne. Essai sur la genèse de l' œuvre en régime naturaliste. Zola, dir. P. Hamon, Genève, Droz, 2009. Voir aussi D. Ferrer, « Quelques remarques sur le couple énonciation-genèse », Texte, $\mathrm{n}^{\circ} 27-28$, Toronto, 2000 , p. $7-24$

10. A. Grésillon, «Langage de l'ébauche : parole intérieure extériorisée », Langages, n 147, septembre 2002. 


\section{Pour une didactique de l'écriture à programme}

L'écriture programmatique, qui dépasse largement une époque et un mouvement artistique, pourra s'incarner dans des avant-textes d'écrivains, forgeant une culture génétique et littéraire, qui montrera aux élèves que leur poétique et leur métatexte induits rejoignent ceux de grands auteurs qu'ils liront peut-être avec une nouvelle connivence. Cette pratique d'écriture, tout juste esquissée ici, plaide en faveur d'un continuum gradué de processus et de compétences entre l'expert, qui les possède et les approfondit, et le débutant qui les construit. Le jeune scripteur stimule d'autant mieux son goût à écrire qu'il a accès à une véritable posture «d'auteur » (Catherine Tauveron), et aux pratiques des écrivains, dans la mesure où elles sont intériorisées dans des situations réelles de production. Les formes d'écriture à programme peuvent développer des compétences en rapport avec la pensée réflexive, l'abstraction et la métacognition, le plaisir de créer aussi, qui ne cessent d'évoluer tant chez le rédacteur débutant que chez l'écrivain, à l'échelle de sa vie d'artiste. L'ébauche du scénario, le dessin de fiction, l'élaboration dialoguée constituent les grandes portes qui ouvrent sur une écriture " expérimentale », fondant sa dynamique créatrice sur la dialectique du cadre programmatique et de sa marge. De fait, la transgression possible du « contrat génétique » passé avec soi, notamment grâce aux échappées du processus rédactionnel qui s'articule au projet, rendrait absurde une dérive applicationniste du «programme » conçu seulement comme un kit d'outillage.

Au contraire, il s'agit toujours d'inventer des situations d'écriture qui permettent à un élève de s'enrichir d'une expérience sensible où le mot «brouillon » hésiterait entre «brouillard et bouillon », selon la belle formule de Philippe Lejeune11 : la dérive de l'idée qui se cherche au fil des écrits, l'opacité et la promesse des mots, le contraire même d'une réponse univoque aux ordres d'une consigne. Le projet didactique d'écriture, venant de l'enseignant, et que doit s'approprier l'élève, peut être enrichi de la projection d'écriture, mouvement dynamique du jeune auteur qui, accompagné de cet enseignant, cherche «sa » poétique. Pour cela, l'élève a besoin de traces du travail de sa pensée, non pour constituer des archives mais pour se créer des outils d'expertise et de régulation de ses propres intentions esthétiques, au moyen de l'autoconsigne verbale, du dessin de fiction ou du plan détaillé.

En menant une réflexion sur les noces possibles de la génétique et de la didactique, cet article a souhaité, par le biais de l'écriture à programme qui déplie l'acte de création dans la durée, construire chez l'élève une mémoire d'encre qui serait « moins une science qu'une méditation et un apprentissage $12 »$. En retour, un autre horizon de recherche, encore lointain, serait de voir si l'étude des écritures à programme in vivo éclairerait les approches in vitro des manuscrits d'écrivains disparus.

\section{BIBLIOGRAPHIE SÉLECTIVE}

Alcorta, M., «Utilisation du brouillon et développement des capacités d'écrit », Revue française de pédagogie, $\mathrm{n}^{\circ} 137$, 2001, p. 95-103.

Bucheton, D., Écriture, réécriture, récits d'adolescents, Berne, Peter Lang, 1995.

David, J., Plane, S. (dir.), L'Apprentissage de l'écriture à l'école et au collège, Paris, PUF, 1996.

Doquet-Lacoste, C., «L'objet insaisissable : l'écriture sur traitement de texte », Genesis, ${ }^{\circ} 27,2006$, p. 35-44.

FABre-Cols, C., Réécrire à l'école et au collège. De l'analyse des brouillons à l'écriture accompagnée, Issy-les-Moulineaux, ESF, 2002.

FAyol, M., Des idées au texte. Psychologie cognitive de la production verbale, orale et écrite, Paris, PUF, 1997.

Garcia-Debanc, C., L'Élève et la production d'écrits, Metz, CASUM, 1990.

«Processus rédactionnels et pédagogie de l'écriture », Pratiques, $\mathrm{n}^{\circ} 49,1986$, p. 23-50.

Gombert, J.-E., Le Développement métalinguistique, Paris, PUF, 1990.

GrÉsILlon, A., « Les manuscrits littéraires : le texte dans tous ses états », Pratiques, n 57,1988 , p. 107-122.

Groupe Eva, Évaluer les écrits des élèves à l'école primaire, Paris, INRP-Hachette Éducation, 1991.

11. P. Lejeune, Les Brouillons de soi, Paris, Éditions du Seuil, 1998, p. 7.

12. Ibid., p. 10. 
De l'évaluation à la réécriture, Paris, INRP-Hachette Éducation, 1996.

Hayes, J.-R., Flower, L. S., « Identifying the organisation of writing processes », dans L. W. Gregg et E. R. Steinberg (dir.), Cognitive processes in writing, Hillsdale, Lawrence Erlbaum, 1980.

Le Goff, F., « Écriture d'invention, réécriture et enseignement de la littérature », thèse de doctorat de l'université de Metz, sous la direction de M. André Petitjean, 2 t., 455 p. et annexes, 2006.

Lumbroso, O., "Esquisse d'un dialogue entre didactique de l'écrit et critique génétique : l'élève "auteur-dessinateur" ", Revue française de pédagogie, $\mathrm{n}^{\circ}$ 159, mai-juin 2007.

«L'écrit d'invention en Seconde : pour un développement de la compétence programmatique », Repères, $\mathrm{n}^{\circ} 40$, décembre 2009.
Oriol-Boyer, C., Critique génétique et didactique de la réécriture. Travailler avec les brouillons d'écrivains, Paris, BertrandLacoste, 2003.

Penloup, M.-C., La Tentation du littéraire, Paris, Didier, 2000.

REPĖRES, «Écrire avec, sur, de la littérature », numéro coordonné par C. Doquet-Lacoste, O. Lumbroso, C. Tauveron, INRP, $\mathrm{n}^{\circ} 40$, décembre 2009.

Reuter, Y., Enseigner et apprendre à écrire. Construire une didactique de l'écriture, Paris, ESF, 1996.

Pratiques, $\mathrm{n}^{\circ}$ 105-106, «La réécriture », 2000.

TAUVERon, C., Vers une écriture littéraire ou comment construire une posture d'auteur à l' école, Paris, Hatier, 2005. 
Olivier Lumbroso est maître de conférences à l'université de Bretagne occidentale et membre de l'équipe Zola (ITEMCNRS-ENS).

Olivier Lumbroso, olivier.lumbroso@bretagne.iufm.fr

\section{Résumés \\ Pour une didactique du prérédactionnel}

Cet article se pose la question suivante : comment aider l'élève à entrer dans l'écriture narrative ? À partir d'expérimentations réalisées à différents niveaux de classe, l'article propose quelques pistes didactiques afin de développer chez l'élève des capacités à programmer l'écrit à l'état natif, et ce en amont du classique premier jet que représente en général le «brouillon ». En effet, que le support soit un croquis, un scénario, un plan détaillé, une liste, ou des autoconsignes, il semble essentiel d'offrir à l'élève un espace du projet d'écriture dans lequel il puisse prévoir ce qu'il veut faire, peut faire et sait faire. Donner à l'élève l'opportunité de forger son propre métatexte génétique semble une voie possible pour développer l'autonomie et la motivation à entrer dans un projet propre et une poïétique à soi. Il apparaît que la compétence programmatique peut déplacer la perspective sur l'écriture en verbalisant explicitement des intentions esthétiques, un souci du lecteur, l'anticipation des structures du texte. La didactique du rédactionnel au cœur des recherches des trente dernières années se voit ainsi complétée par une didactique du prérédactionnel qui nourrit efficacement la préparation du texte.

The question is: how can we help schoolchildren undertake narrative writing? Based on experiments carried out at different class levels, this article suggests a few didactic possibilities for developing schoolchildren's capacities to program writing at a nascent stage - upstream so to speak - of the classic first draft, usually considered the "rough draft". Indeed, whether the support be a sketch, a scenario, a detailed outline, a list, or instructions for oneself, it seems essential to offer schoolchildren a writing project space in which they can anticipate what they wished to do, can do and know how to do. To give a schoolchild the opportunity of creating his/her genetic metatext seems a possible way to develop the necessary autonomy and motivation to undertake a personal project and one's own poïetic. It seems that programmatic skills can shift the perspective on writing by explicitly putting into words: esthetic intentions, concern for the reader, the anticipation of the text's structures. Editorial didactics, at the heart of the last thirty years' research, is thus complemented by pre-editorial didactics which effectively enriches the preparation of the text.
Der Beitrag stellt folgende Frage: Wie kann man einem Schüler helfen, der lernen soll, eine Erzählung zu schreiben? Ausgehend von Experimenten, die in verschiedenen Klassen durchgeführt wurden, schlägt der Artikel didaktische Wege vor, um den Schüler fähig zu machen, sein Schreibprogramm sehr früh zu entwickeln, und zwar schon bevor er den sogenannten ,ersten Wurf" zu Papier bringt. Ob dieses Programm die Form einer Skizze, eines Szenarios, eines detaillierten Plans, einer Liste, oder von an sich selbst gerichteten Schreibbefehlen annimmt, das Wichtigste dabei scheint, dem Schüler einen Raum zu bieten, in dem er aufschreiben kann, was er tun will, was er tun kann und was er zu tun weiß. Dem Schüler Gelegenheit zu geben, seinen eigenen genetischen Metatext zu entwickeln, öffnet ihm einen Weg, um sich durchaus selbständig für ein Schreibprojekt zu motivieren und seine eigene Schreibpraxis zu fördern. Diese „Programmierungskompetenz“ scheint sich auch auf die Schreibkompetenz selbst auszuwirken, insofern sie aesthetische oder auf den Leser gezielte Absichten, sowie auch künftige Textstrukturen ausdrücklich formuliert. Die Didaktik des Aufsatzschreibens, die seit dreißig Jahren im Mittelpunkt steht, wird so ergänzt durch eine Didaktik des Planens, die auf effiziente Weise die Vorbereitung des Textes bereichert.

Este artículo se plantea el siguiente interrogante: ¿cómo ayudar al alumno a entrar en la escritura narrativa? A partir de experimentaciones realizadas a distintos niveles de clase, el artículo propone algunas pistas didácticas para desarrollar en el alumno la capacidad de programar la escritura en su estado nativo, previo a la clásica primera versión representada en general por el "borrador". En efecto, sea cual fuere el soporte -un croquis, un guión, un plan detallado, una lista o una serie de autoconsignas-, resulta esencial ofrecer al alumno un espacio de proyecto de escritura, en el cual éste pueda prever lo que quiere hacer, puede hacer y sabe hacer. Ofrecer al alumno la oportunidad de forjar su propio metatexto genético aparece como una posible vía para desarrollar la autonomía y la motivación para entrar en un proyecto y una poética propia. Se pone de manifiesto la capacidad de la competencia programática a desplazar la perspectiva hacia la escritura verbalizando explícitamente las intenciones estéticas, la preocupación por el lector, la anticipación de las estructuras del texto. La didáctica de lo redaccional, predominante en las investigaciones de los últimos treinta años, se conjuga así con una didáctica de lo preredaccional que sedimenta eficazmente la preparación del texto. 
Coloca-se a seguinte questão: como ajudar o aluno a penetrar na escrita narrativa? A partir de experimentações realizadas a diversos níveis de ensino, o artigo propõe algumas pistas didácticas para desenvolver no aluno a capacidade de programar o escrito em estado nato, bem antes do clássico primeiro jacto que produz o "rascunho". Com efeito, quer o suporte seja um esboço, um cenário, um plano detalhado, uma lista, ou anotações breves, parece essencial oferecer ao aluno um espaço, dentro do projecto de escrita, no qual possa prever aquilo que deseja, pode e sabe fazer. Dar ao aluno a oportunidade de forjar o seu próprio metatexto genético parece uma via possível para desenvolver a autonomia e a motivação de entrar num projecto próprio, com a sua própria poética. A competência programante pode deslocar a perspectiva sobre a escrita por uma verbalização explícita de intenções estéticas, de uma preocupação com o leitor, da antevisão das estruturas do texto. A didáctica redaccional, central às pesquisas dos trinta últimos anos, vê-se assim completada por uma didáctica do pré-redaccional que nutre com eficácia a preparação do texto.

Come aiutare l'allievo ad introdursi nella scrittura narrativa ? È la domanda che si pone quest'articolo. Partendo da esperimenti realizzati in diversi stadî dell'insegnamento scolastico, tale riflessione propone alcuni percorsi didattici che permettono di approfondire nell'allievo la capacità di pianificazione della scrittura sin nel suo stadio iniziale, ancor prima cioè della classica minuta iniziale rappresentata in generale dalla "brutta copia". A prescindere dal supporto, che si tratti di uno schizzo, di un canovaccio, di uno schema dettagliato di lavoro, di una lista, o di auto-indicazioni di scrittura, appare infatti essenziale offrire all'allievo le linee di un programma redazionale all'interno del quale egli possa prevedere ciò che vuole, può e sa esprimere. Dare all'allievo l'opportunità di forgiare il proprio metatesto genetico può, a quanto pare, essere una via possibile per sviluppare l'autonomia e la motivazione ad avviare un progetto ed una poietica proprî. Sembra, difatti, che la competenza programmatica possa far variare il rapporto con la scrittura, nella misura in cui tale competenza implicherà una dichiarazione esplicita di intenzioni estetiche, una certa attenzione al lettore, oltreché l'anticipazione delle future strutture testuali. La didattica applicata all'aspetto propriamente redazionale, al centro delle ricerche durante gli ultimi trent'anni, si vede così completata da una didattica legata alla fase pre-redazionale, la quale nutre efficacemente l'allestimento del testo. 